\title{
Personal gewinnen und halten - die Suche nach dem karierten Maiglöckchen
}

\author{
Eva-Sabine Roßwaag
}

Online publiziert: 24. Oktober 2012

(C) Springer-Verlag Wien 2012

Ein zentrales Thema in der Gesundheits- und Sozialwirtschaft ist derzeit die aufgrund von fortschreitendem Ressourcenabbau kontinuierliche Leistungsverdichtung, was eine Erhöhung des Arbeitspensums zur Folge hat. Begünstigt durch die demografische Entwicklung und den damit kontinuierlichen Anstieg des Durchschnittsalters nicht nur der Klienten sondern auch der Mitarbeitenden werden innovative Ansätze zur Gewinnung und Bindung von Mitarbeitenden benötigt.

Ein strategisches Instrument hierzu bildet die Entwicklung eines Personalmarketingkonzeptes, das die frühzeitige Nachfolgeplanung berücksichtigt sowie die darauf basierende Herausbildung einer Personalentwicklung. Gefordert ist also die Implementierung eines innovativen Personalmarketingsystems.

Personalmarketing als Konzept trägt dafür Sorge, dass Unternehmen ihre Personalpolitik an den Bedürfnissen und Wünschen qualifizierter Fach- und Führungskräfte ausrich- ten, um aktuelle Mitarbeitende zu binden, zu entwickeln und potenzielle zu gewinnen. Die Gewinnung qualifizierter Fach- und Führungskräfte wird jetzt und zukünftig an die Güte des Personalmarketingsystems gekoppelt sein und setzt ein aktives Werben sozialer Organisation um Mitarbeitende voraus.

Wesentlich für den langfristigen Erfolg ist die Entwicklung einer werteorientierten Unternehmenskultur, in der Glaubwürdigkeit, Fairness und Respekt, sowie die Gesundheit am Arbeitsplatz eine tragende Rolle spielen. Langfristig Erfolg auf dem umkämpften Arbeitsmarkt für qualifizierte Fach- und Führungskräfte werden nur Unternehmen haben, die nach ganzheitlichen, in der Gesamtstrategie des Unternehmens integrierten und innovativen Konzepten im Personalmarketing arbeiten. Insbesondere der Aufbau und die Kommunikation einer attraktiven Arbeitgebermarke stellt hierbei eine entscheidende Maßnahme dar.

E.-S. Roßwaag $(\triangle)$

contec $\mathrm{GmbH}$, Bochum, Deutschland 